\title{
Wnt6 contributes tumorigenesis and development of colon cancer via its effects on cell proliferation, apoptosis, cell-cycle and migration
}

\author{
XIAO-LI ZHENG and HONG-GANG YU \\ Department of Gastroenterology, Renmin Hospital of Wuhan University, Wuhan, Hubei 430060, P.R. China
}

Received January 13, 2017; Accepted May 3, 2018

DOI: $10.3892 / \mathrm{ol} .2018 .8729$

\begin{abstract}
Wnt proteins have been reported to contribute to the progression of various types of cancer. Wnt6 is a member of the Wnt family and may promote tumorigenesis in gastrointestinal cancer and cervical cancer. In the present study, the expression of Wnt6 in human colon cancer cell lines was evaluated, in order to investigate the role of Wnt6 in the development of colon cancer. Additionally, the effects of Wnt6 upregulation or downregulation on proliferation, apoptosis, cell cycle and cell migration of colon cancer cells have been investigated. Furthermore, western blot analysis was employed to evaluate the expression of Wnt6, B-cell lymphoma 2-associated $\mathrm{X}$ protein (Bax), caspase-3 and matrix metalloproteinase (MMP)2. The results of the present study demonstrated that the expression of Wnt6 was increased in HCT116 and SW480 cells compared with the remaining colon cancer cell lines. Furthermore, overexpression Wnt6 resulting from transfection of pGPU6/GFP/Neo-Wnt6-Homo-1 plasmid promoted the proliferation, cell cycle and migration of HCT116 and SW480 cells, but inhibited cell apoptosis in vitro. The expression of caspase-3 and MMP2 was increased, whereas the expression of Bax was decreased in response to upregulation of Wnt6. These results suggested that Wnt6 may serve a vital function in the development of colon cancer.
\end{abstract}

\section{Introduction}

Colon cancer is a common malignant tumor of the gastrointestinal tract and is the fourth leading cause of cancer-associated mortality (1). The 5-year survival rate of patients with colon cancer is $50 \%(1,2)$. The onset of colon cancer is associated with genetic and environmental factors (e.g., exposure to carcinogens and smoking) (3). Inactivation of tumor suppressor

Correspondence to: $\mathrm{Dr}$ Hong-Gang Yu, Department of Gastroenterology, Renmin Hospital of Wuhan University, 99 Zhangzhidong Road, Wuchang, Wuhan, Hubei 430060, P.R. China E-mail: honggyu16@sina.com

Key words: Wnt6, colon cancer, Wnt signaling pathway, proliferation, cell cycle, apoptosis, migration genes and mutation of oncogenes lead to the development of malignant tumors (3). Previous studies investigated the effect of drugs on the proliferation, adhesion, invasion and migration of colon cancer cells, and the underlying molecular mechanisms of drug resistance in colon cancer (4-9).

The Wnt signaling pathway regulates diverse developmental processes, including cell adhesion, proliferation, differentiation, migration and apoptosis (10). Previous studies have demonstrated that numerous types of cancer, including melanoma, hepatocarcinoma, gastrointestinal, breast and ovarian cancer (11), are associated with abnormal Wnt signaling pathway. Abnormal activation of Wnt signaling pathway has been reported in colorectal cancer $(12,13)$. Wnt family of proteins includes at least 19 secreted-type glycoproteins with conserved 22-24 cysteine residues, and serves vital functions in carcinogenesis and embryogenesis (14). Previous studies have reported that Wnt6 is upregulated in gastrointestinal cancer and cervical cancer, and overexpression of Wnt6 promotes physiological or pathological processes via activation of Wnt/ $\beta$-catenin signaling pathway in various cancer cell lines (15-18). Wnt6 is highly expressed in colorectal adenoma and may be associated with increased risk of colorectal cancer (19). However, the role of Wnt6 in occurrence, progression and metastasis of colon cancer remains unclear.

In the present study, the expression of Wnt6 was evaluated in colon cancer cell lines (LoVo, SW480, HCT116, SW620 and HT29). The effects of overexpression and knockdown of Wnt6 on proliferation, cell cycle and apoptosis of colon cancer cells were investigated. The aim of the present study was to investigate the function of Wnt6 in tumorigenesis and progression of colon cancer and provide the basis for a novel therapeutic target in the treatment of colon cancer.

\section{Materials and methods}

Cell culture. Wnt6 high expression cell lines were selected from five human colon cancer cell lines (LoVo, SW480, HCT116, SW620 and HT29). Cells were maintained in Dulbecco's modified Eagle's medium (DMEM; Gibco; Thermo Fisher Scientific, Inc., Waltham, MA, USA) supplemented with $10 \%$ fetal bovine serum (FBS, Gibco; Thermo Fisher Scientific, Inc.), $100 \mathrm{U} / \mathrm{ml}$ of penicillin and $100 \mu \mathrm{g} / \mathrm{ml}$ of streptomycin, and were cultured at $37^{\circ} \mathrm{C}$ in a humidified atmosphere containing $5 \% \mathrm{CO}_{2}$. 
Reverse transcription-quantitative polymerase chain reaction $(R T-q P C R)$. Total RNA was extracted from cells using TRIzol reagent (Ambion; Thermo Fisher Scientific, Inc.). RNA was reverse-transcribed into cDNA using PrimeScript ${ }^{\mathrm{TM}}$ 1st Strand cDNA Synthesis kit (Takara Biotechnology Co., Ltd., Dalian, China), and qPCR was performed using a KAPA SYBR Green qPCR kit (Kapa Biosystems, Inc., Wilmington, MA, USA). The primer sequences for Wnt6 were as follows: 5'-CGG AAGTGGTGGCAGAG-3' (forward) and 5'-CAGGATGCG TCCAAAGG-3' (reverse). The primer sequences for $\beta$-actin were as follows: 5'-ACACTGTGCCCATCTACG-3' (forward) and 5'-TGTCACGCACGATTTCC-3' (reverse). Each reaction (20 $\mu \mathrm{l}$ total volume) contained $10 \mu \mathrm{l} \mathrm{SYBR}, 0.40 \mu \mathrm{mol} / 1$ each primer and $0.2 \pm 0.02 \mu \mathrm{g}$ cDNA template. The thermocycling conditions were as follows: Pre-denaturation at $95^{\circ} \mathrm{C}$ for $3 \mathrm{~min}$, followed by 40 cycles of denaturation at $95^{\circ} \mathrm{C}$ for $5 \mathrm{sec}$, annealing at $60^{\circ} \mathrm{C}$ for $20 \mathrm{sec}$ and elongation at $72^{\circ} \mathrm{C}$ for $20 \mathrm{sec}$. The threshold cycle $(\mathrm{Ct})$ was determined for each reaction by using the $2^{-\Delta \Delta \mathrm{Cq}}$ method, which generated $\mathrm{Ct}$ values for each gene of interest normalized to the endogenous control gene ( $\beta$-actin) (20). For each group, three replicates of each measurement were performed.

Cell transfection. Cells (HTC116 or SW480) in $1 \times 10^{5}$ cells $/ \mathrm{ml}$ were seeded in 6-well plates and cultured until cells reached confluency (70-80\%). Cells were then transfected with $800 \mathrm{ng} /$ well of plasmids using Lipofectamine ${ }^{\circledR} 2000$ (Invitrogen; Thermo Fisher Scientific, Inc.), according to the manufacturer's protocol. The primers for Wnt6 were as follows: 5'-CACCCTGCCGCCCTTACCCTCC-3' (forward) and 5'-GATCCGGGTCACAGGCAGAGGC-3' (reverse). The corresponding cDNAs were inserted into the pGPU6/green fluorescent protein (GFP)/Neo vector to construct the recombinant pGPU6/GFP/Neo-Wnt6-Homo-1 plasmid, to overexpress Wnt6 (GenePharma Shanghai, China). An empty vector (EV) was used as a negative control. Additionally, specific short hairpin (sh)RNA-expressing vectors were employed to knockdown Wnt6. Negative control (NC) pGPU6/GFP/Neo-shNC (target sequence, GTTCTCCGAACGTGTCACGT) and pGPU6/GFP/Neo-Wnt6-Homo-A (target sequence, AAG TGGTGGCAGAGCTAGCTC) vectors were obtained from Shanghai GenePharma Co., Ltd. (Shanghai, China). Untransfected HCT116 or SW480 cells consider as control group.

MTT assay. Cell proliferation was evaluated using an MTT assay. HCT116 and SW480 cells were seeded in 96-well plates and transfected with expression or empty vector with $1 \times 10^{4}$ cells in each well. At indicated timepoints $(24,48$ and $72 \mathrm{~h}$ ), $20 \mu \mathrm{l}$ MTT (5 mg/ml; Bioswamp, Wuhan, China) was added to each culture prior to incubation at $37^{\circ} \mathrm{C}$ for an additional $4 \mathrm{~h}$. Then, DMEM medium was removed and $150 \mu \mathrm{l}$ dimethylsulfoxide was added to each well and mixed for $10 \mathrm{~min}$. Absorbance was read at a wavelength of $490 \mathrm{~nm}$.

Flow cytometric analysis of apoptosis. Apoptosis was detected by double staining with Annexin V-fluorescein isothiocyanate (FITC)/propidium iodide (PI) (BDBiosciences, Franklin Lakes, NJ, USA), according to the manufacturer's protocol. At $48 \mathrm{~h}$ post-transfection, HCT116 and SW480 cells $\left(1 \times 10^{6}\right.$ cells $\left./ \mathrm{ml}\right)$ were washed three times with ice-cold PBS and incubated for $30 \mathrm{~min}$ at $4^{\circ} \mathrm{C}$ in the dark in $200 \mu \mathrm{l}$ binding buffer containing $10 \mu \mathrm{l}$ Annexin V-FITC and $10 \mu \mathrm{l}$ PI. Apoptotic cells were analyzed using a flow cytometer (Beckman Coulter, Inc., Brea, CA, USA) and Cytomics FC 500 MCL with CXP software 5.0 network (Beckman Coulter, Inc.).

Analysis of cell cycle. Cell cycle was analyzed using flow cytometry. At 48 h post-transfection, HCT116 and SW480 cells in $1 \times 10^{6}$ cells $/ \mathrm{ml}$ were washed with ice-cold PBS and then fixed with $70 \%$ ethanol at $4^{\circ} \mathrm{C}$ for $1 \mathrm{~h}$. Following washing with PBS, cells were stained with PI [10 mM Tris ( $\mathrm{pH} 7.0), 0.1 \%$ NP-40, $1 \mathrm{mM} \mathrm{NaCl}, 0.7 \mu \mathrm{g} / \mathrm{ml}$ ribonuclease A and $5 \mu \mathrm{g} / \mathrm{ml}$ $\mathrm{PI}$ ] for $30 \mathrm{~min}$ in the dark. Cell cycle analysis was performed using a flow cytometer and Cytomics FC 500 MCL with CXP 5.0 software.

Cell migration assay. Cell migration were assessed using Transwell assays. HCT116 and SW480 cells $\left(1 \times 10^{5}\right.$ cells $\left./ \mathrm{ml}\right)$ were starved in serum-free DMEM medium for 24 At 48 h, cells were fixed for $10 \mathrm{~min}$ using $4 \%$ paraformaldehyde (Bioswamp; Wuhan Beinglay Biological Technology Co., Wuhan, China) and incubated with $0.5 \%$ crystal violet (Bioswamp; Wuhan Beinglay Biological Technology Co.) for $30 \mathrm{~min}$. A total of $100 \mu \mathrm{l}$ cell suspension was seeded into transwell chambers with $8 \mu \mathrm{m}$ prore polycarbonate membrane insert (Corning Incorporated, Corning, NY, USA). A total of $600 \mu \mathrm{l}$ RPMI-1640 medium (Beijing Solarbio Science \& Technology, Co., Ltd., Beijing, China) containing 20\% FBS was added in lower chambers. Following incubation for $24 \mathrm{~h}$ in transwell chamber, cells remaining on the upper membrane were removed carefully with a cotton swab. Stained cells were counted in three fields using a light microscope (Nikon Corporation, Tokyo, Japan; magnification, x200).

Western blot analysis. Western blot analysis was performed as previously described (21). Antibodies against Wnt6 (cat. no. ab50030, 1:500 dilution; Abcam, Cambridge, UK), B-cell lymphoma 2 (Bcl-2)-associated X protein (Bax) (cat. no. ab32503, 1:2,000 dilution; Abcam), caspase-3 (cat. no. ab32351, 1:2,000 dilution; Abcam), matrix metalloproteinase (MMP)2 (cat. no. ab37150, 1:1,000 dilution; Abcam) and $\beta$-actin (cat. no. 49675, 1:1,000 dilution; Cell Signaling Technology, Inc., Danvers, MA, USA) were used. HCT116 and SW480 cells were washed twice with PBS and homogenized in radioimmunoprecipitation assay lysis buffer (Beyotime Institute of Biotechnology, Haimen, China) containing protease inhibitor (cat. no. 58715; Cell Signaling Technology) and centrifuged at $12,000 \mathrm{xg}$ for $15 \mathrm{~min}$ at $4^{\circ} \mathrm{C}$. The concentration of the proteins was measured using a bicinchoninic acid assay kit (cat. no. P0011; Beyotime Institute of Biotechnology). A total of $30 \mu \mathrm{g}$ proteins were separated by $10 \%$ SDS-PAGE and transferred onto a polyvinylidene difluoride membrane (Merck KGaA, Darmstadt, Germany). The membranes were blocked with 5\% skim milk for $2 \mathrm{~h}$ at room temperature in Tris-buffered saline. Then, the membranes were incubated with primary antibodies overnight at $4^{\circ} \mathrm{C}$. Anti- $\beta$-actin antibody was selected as internal reference. Then, the membranes were washed with Tris-buffered saline and incubated in biotinylated goat IgG conjugated to horseradish 
A

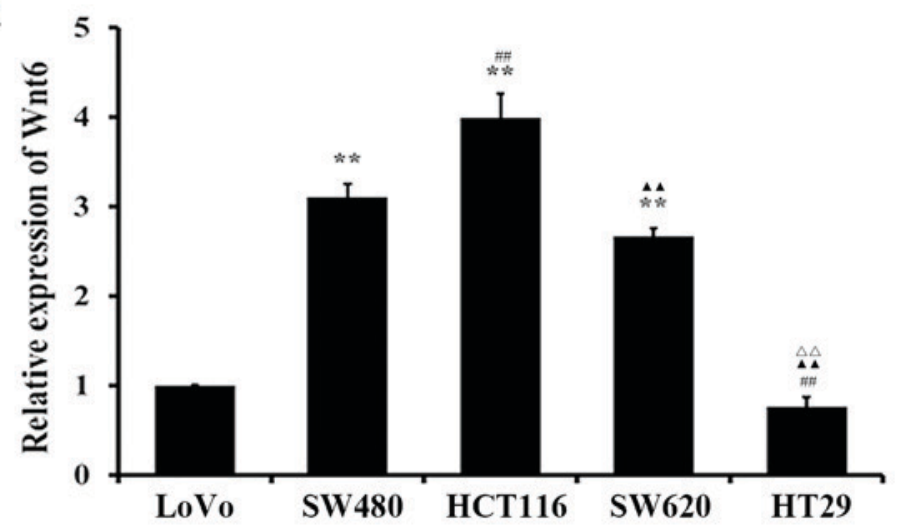

B

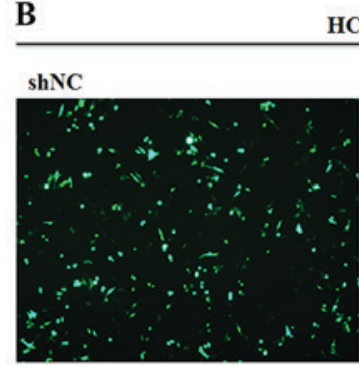

EV
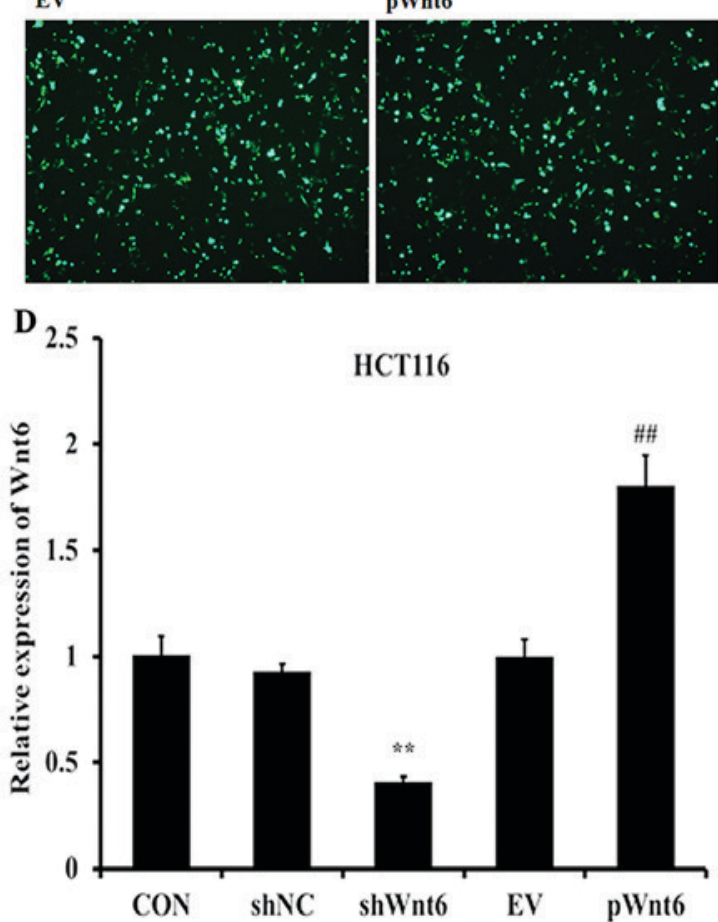

C Sw480

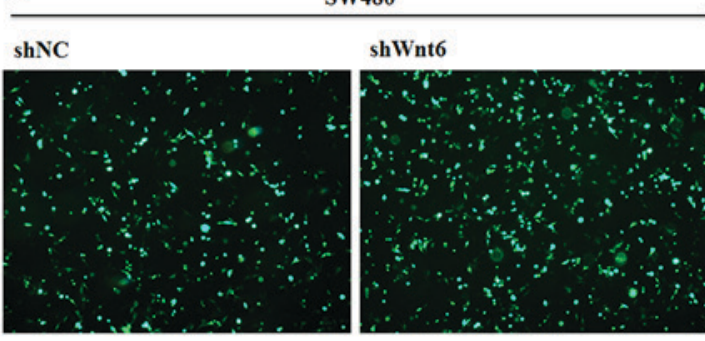

EV

pWnt6
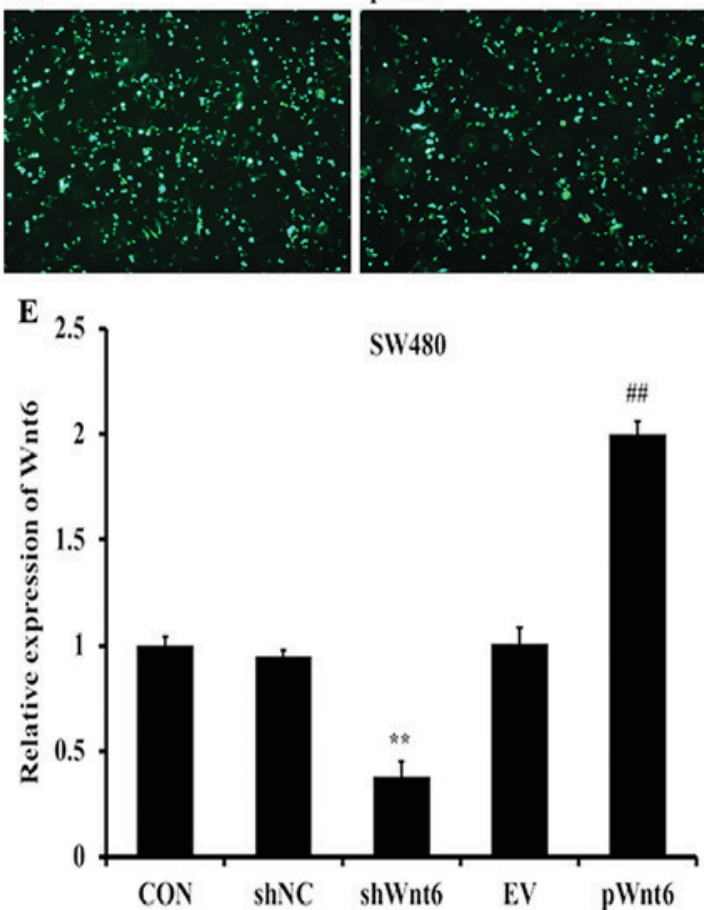

Figure 1. Expression of Wnt6 in human colon cancer cell lines. (A) RT-qPCR analysis of the expression levels of Wnt6 in colon cancer cells (LoVo, SW480, HCT116, SW620 and HT29). ${ }^{* *} \mathrm{P}<0.01$ vs. LoVo; ${ }^{\# \#} \mathrm{P}<0.01$ vs. SW480; ${ }^{\Delta}{ }^{\wedge} \mathrm{P}<0.01$ vs. HCT116; ${ }^{\Delta} \mathrm{P}<0.01$ vs. SW620. HCT116 cells and SW480 cell were transfected with The shNC, shWnt6, EV or pWnt6 were successfully transfected into (B) HCT116 cells and (C) SW480 cell and detected using fluorescence microscope. The expression levels of Wnt6 in (D) HCT116 cells and (E) SW480 cells transfected with shNC, shWnt6, EV or pWnt6 as detected using RT-qPCR. ${ }^{* * *} \mathrm{P}<0.01$ vs. shNC; ${ }^{\# \#} \mathrm{P}<0.01$ vs. shWnt6. RT-qPCR, reverse transcription-quantitative polymerase chain reaction; sh, short hairpin; NC, negative control; EV, empty vector; p, plasmid; CON, control.

peroxidase (HRP) secondary antibody (cat. no. ab7090, Abcam) for $2 \mathrm{~h}$ at room temperature. Immunoreactivity was visualized by colorimetric reaction using an enhanced chemiluminesence substrate buffer (Merck KgaA). Membranes were scanned with Gel Doz EZ imager (Bio-Rad Laboratories, Inc., Hercules, CA, USA). Bans were quantified using Quantity One 5.0 software (Bio-Rad Laboratories, Inc.).
Statistical analysis. Data were analyzed using SPSS software (version 18.0; SPSS, Inc., Chicago, IL, USA). The relevant data are expressed as the mean \pm standard error of the mean. Statistical analysis was performed using one-way analysis of variance followed by Duncan's multiple range test. $\mathrm{P}<0.05$ was considered to indicate a statistically significant difference. 


\section{Results}

Expression of Wht6 in human colon cancer cell lines. RT-qPCR was employed to evaluate the expression levels of Wnt6 in human colon cancer cells. The results demonstrated that HCT116 and SW480 cells exhibited increased expression levels of Wnt6 (Fig. 1A). Therefore, we selected HCT116 and SW480 cells lines in the further studies, to explore the effect of Wnt6 expression on colon cancer. Additionally, pGPU6/GFP/Neo-Wnt6-Homo-1 plasmid was constructed to assess the effects of overexpression of Wnt6, whereas specific shRNA-expressing vectors were employed to knockdown Wnt6. Fig. 1B and $\mathrm{C}$ demonstrate the transfection efficiency of vectors using HCT116 and SW480 cells. As presented in Fig. 1D and E, shWnt6 (a plasmid carrying shRNA targeting Wnt6) significantly reduced the expression of Wnt6, whereas pWnt6 (Wnt6 overexpression plasmid) significantly increased the expression of Wnt6 in HCT116 and SW480 cells.

Overexpression of Wnt6 induced colon cancer cell proliferation. Cell proliferation was evaluated using an MTT assay. Cells were divided into the following groups: Control, shNC (transfection with negative control), shWnt6 (transfection with shWnt6), EV (transfection with empty vector) and shWnt6+pWnt6 (combined transfection with shWnt6 and pWnt6). The results demonstrated that cell proliferation was decreased in the shWnt6 group compared with that of the shNC group at 24, 48 and 72 h (Fig. 2A and B), indicating downregulation of Wnt6 inhibited the proliferation of HTC116 and SW480. Cell viability was significantly increased in the shWnt6+pWnt6 group in a time-dependent manner compared with the shWnt6 group (Fig. 2A and B). Therefore, knockdown of Wnt6 decreased cell proliferation and transfection with pWnt6 reversed this effect in HCT116 and SW480 cells.

Overexpression of Wnt6 inhibits the apoptosis of colon cancer cell. Cell apoptosis was evaluated using Annexin V-FITC/PI staining and flow cytometry. As presented in Fig. 3A and B, the percentage of apoptotic cells was significantly increased in the shWnt6 group compared with that of the shNC group. However, shWnt6+pWnt6 group exhibited decreased apoptosis compared with that of shWnt6 group. Knockdown of Wnt6 increased apoptosis and transfection with pWnt6 reversed this effect in HCT116 and SW480 cells.

Overexpression of Wnt6 promotes colon cancer cell cycle. The effect of Wnt6 on cell cycle was evaluated using flow cytometry. As presented in Fig. 4A and B, cells transfected with shWnt6 exhibited a significant $\mathrm{G}_{0}-\mathrm{G}_{1}$ cell cycle arrest accompanied with a reduction of cell numbers in $\mathrm{S}$-phase, which was reversed in response to transfection with pWnt6. These results suggest that knockdown of Wnt6 may induce cell cycle arrest in $\mathrm{G}_{0}-\mathrm{G}_{1}$ phase in colon cancer cell lines and transfection with pWnt6 reversed this effect.

Overexpression of Wnt6 induces the migration of colon cancer cell. Cell migration was evaluated using Transwell assays. As presented in Fig. 5A and B, transfection with shWnt6 significantly suppressed the migration of cells compared with that of the shNC group, whereas shWnt6+pWnt6 group exhibited
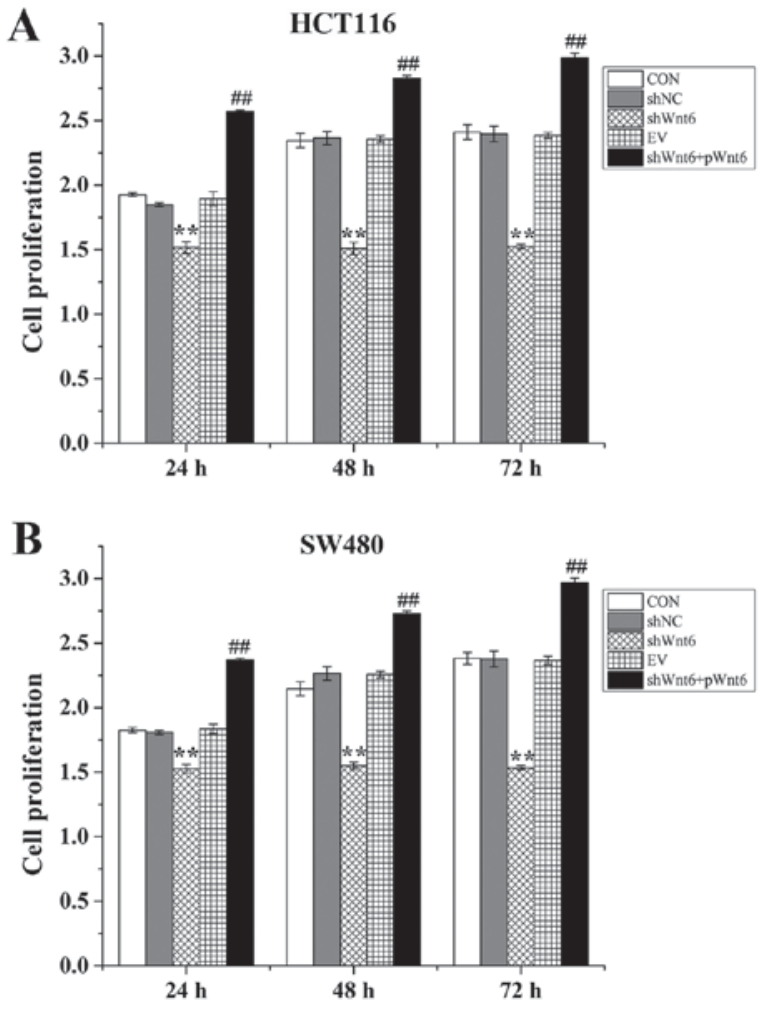

Figure 2. Wnt6 promotes cell proliferation in HCT116 and SW480 cells Cell proliferation was evaluated using an MTT assay. The proliferation of (A) HCT116 and (B) SW480 cells in CON, shNC, shWnt6, EV and shWnt6 groups. ${ }^{* *} \mathrm{P}<0.01$ vs. shNC; ${ }^{\# \#} \mathrm{P}<0.01$ vs. shWnt6. sh, short hairpin; NC, negative control; EV, empty vector; p, plasmid; CON, control.

increased numbers of migrated cells. Knockdown of Wnt6 decreased the migratory ability of cells and transfection with pWnt6 reversed this effect in HCT116 and SW480 cells.

Overexpression of Wnt6 effects on the expression of apoptosis-associated proteins. The expression of Bax, caspase-3 and MMP2 was assessed using western blot analysis. The results confirmed that shWnt6-transfected cells exhibited decreased expression levels of Wnt6 compared with that of the shNC group, whereas the shWnt6+pWnt6 group exhibited increased expression levels of Wnt6 compared with that of the shNC group (Fig. 6A and B). Additionally, the expression levels of caspase- 3 and MMP2 were decreased, whereas the expression levels of Bax were increased in response to shWnt6 (Fig. 6). The expression of caspase-3 and MMP2 was increased, whereas the expression of Bax was decreased following overexpression of Wnt6 (achieved by pWnt6) (Fig. 6A and B).

\section{Discussion}

The Wnt/ $\beta$-catenin signaling pathway is triggered by a series of signaling cascade reactions, thus leading to transcription of target genes in the nucleus (10). The Wnt signaling pathway is involved in cell proliferation, apoptosis and epithelial-mesenchymal transition (EMT) (22). Additionally, previous studies demonstrated that aberrant Wnt signaling pathway promotes cell proliferation and tumorigenesis (23) in various types of cancer, including gastrointestinal (24), breast (25), kidney (26), pancreatic (27), prostate cancer (28), melanoma (29) and 
A
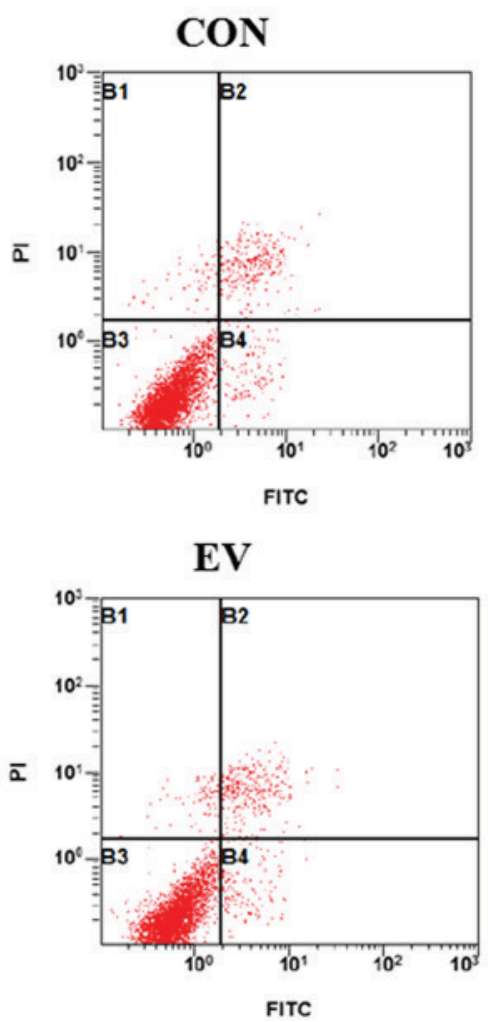

B

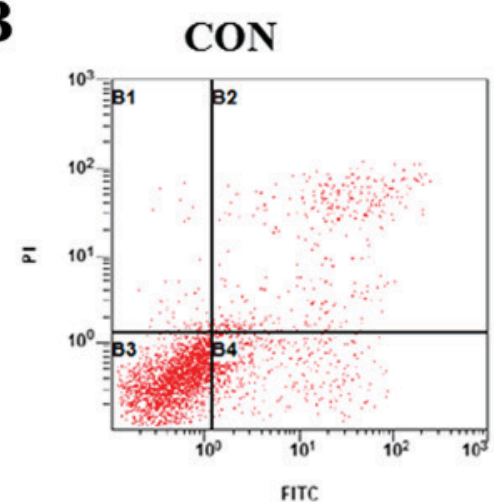

EV

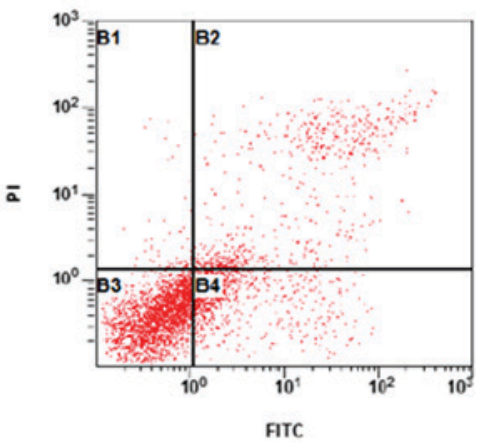

shNC

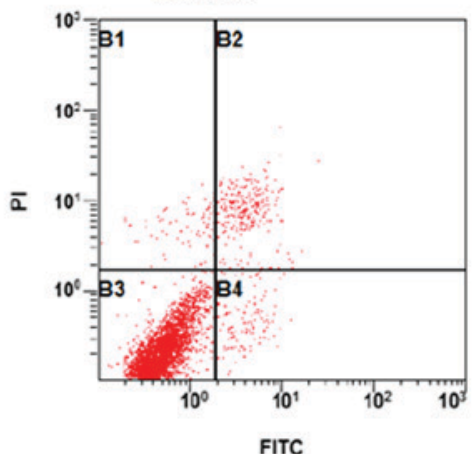

shWnt6+pWnt6

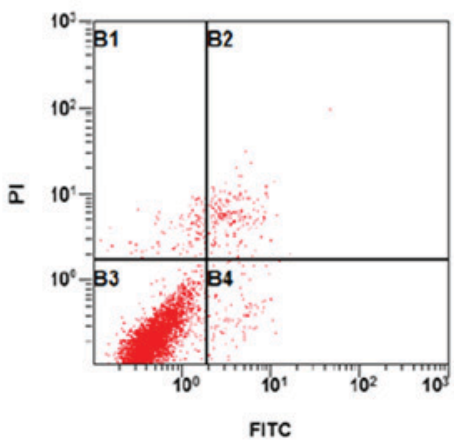

shNC

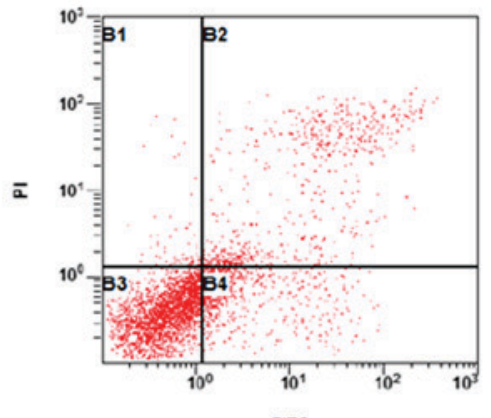

FIIC

shWnt6+pWnt6

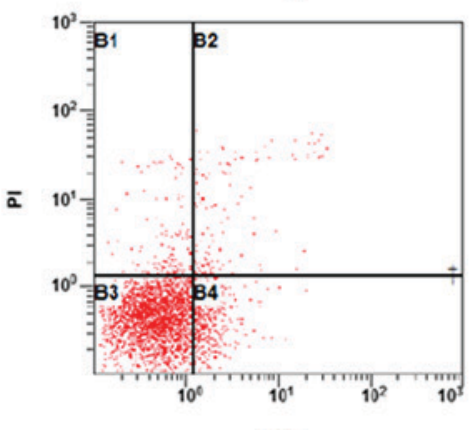

shWnt6
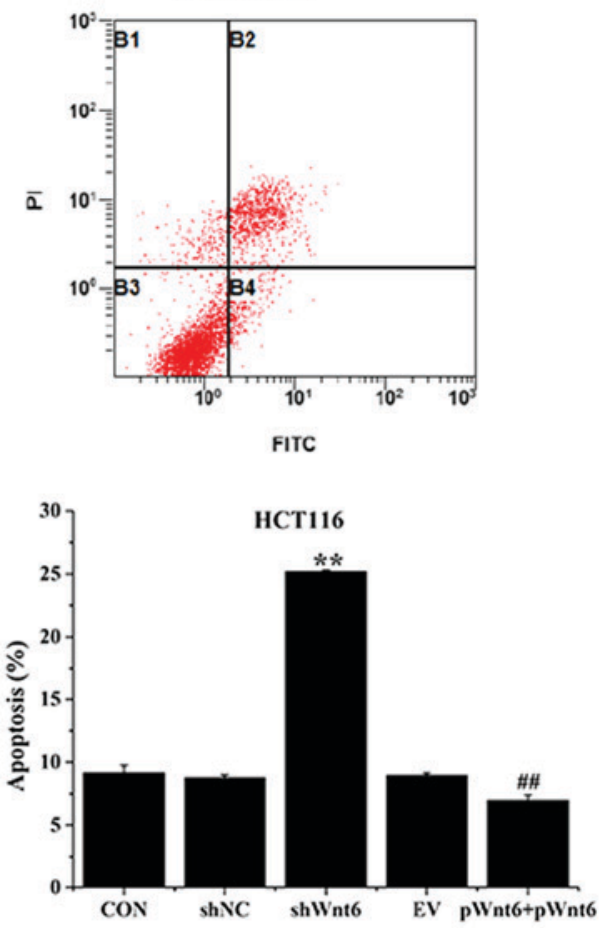

shWnt6

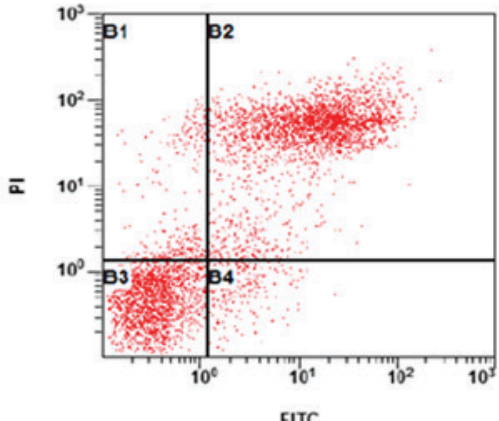

SW480

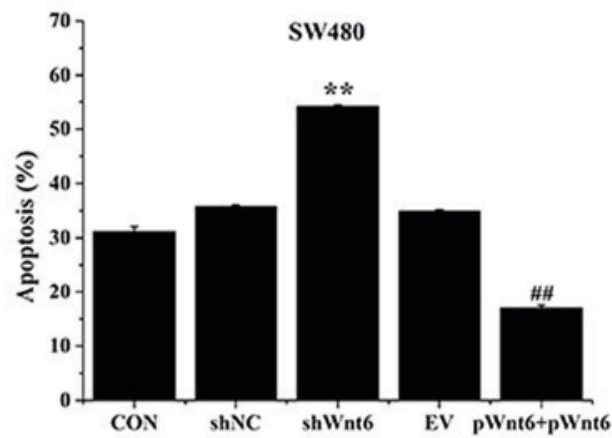

Figure 3. Wnt6 decreases apoptosis in HCT116 and SW480 cells. The percentage of apoptotic cells in (A) HCT116 and (B) SW480 cells in CON, shNC, shWnt6, EV and shWnt6 groups was evaluated using flow cytometry. ${ }^{* *} \mathrm{P}<0.01$ vs. shNC; ${ }^{* \#} \mathrm{P}<0.01$ vs. shWnt6. sh, short hairpin; NC, negative control; EV, empty vector; p, plasmid; CON, control.

osteosarcoma (30). Wnt6 is a member of the Wnt protein family that has been reported to be involved various types of cancer $(19,31)$. However, the function of Wnt6 in colon cancer remains unclear.
In the present study, the expression of Wnt6 was increased in HCT116 and SW480 cells compared with LoVo and HT29 cells. Therefore, HCT116 and SW480 cells were selected for subsequent experiments. The expression pattern of Wnt6 may 
A
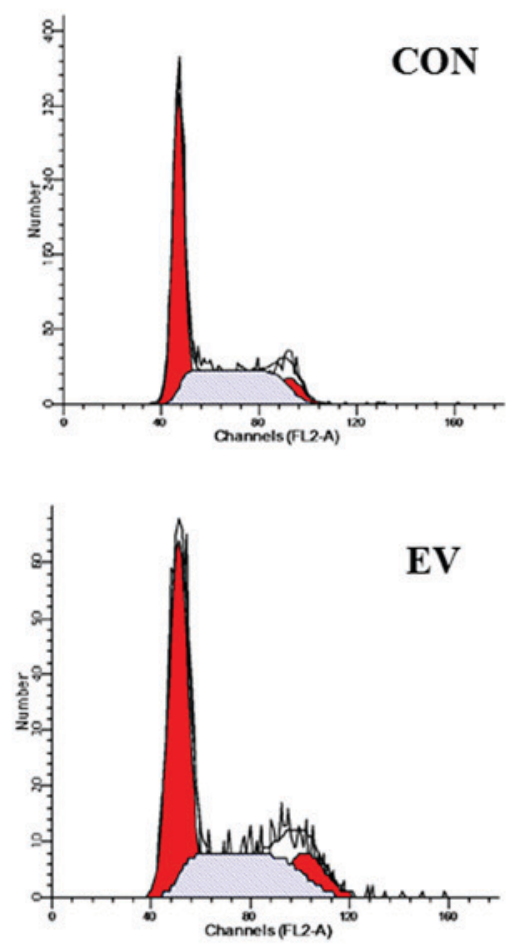

B
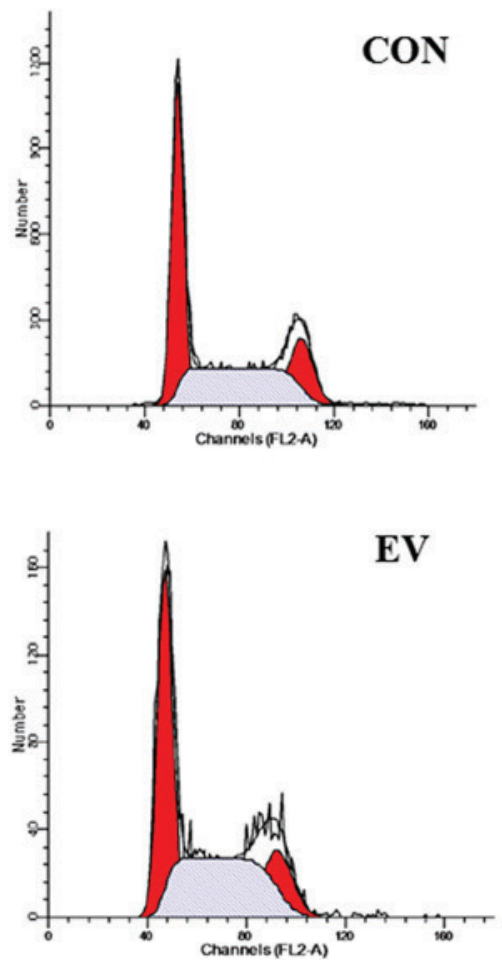
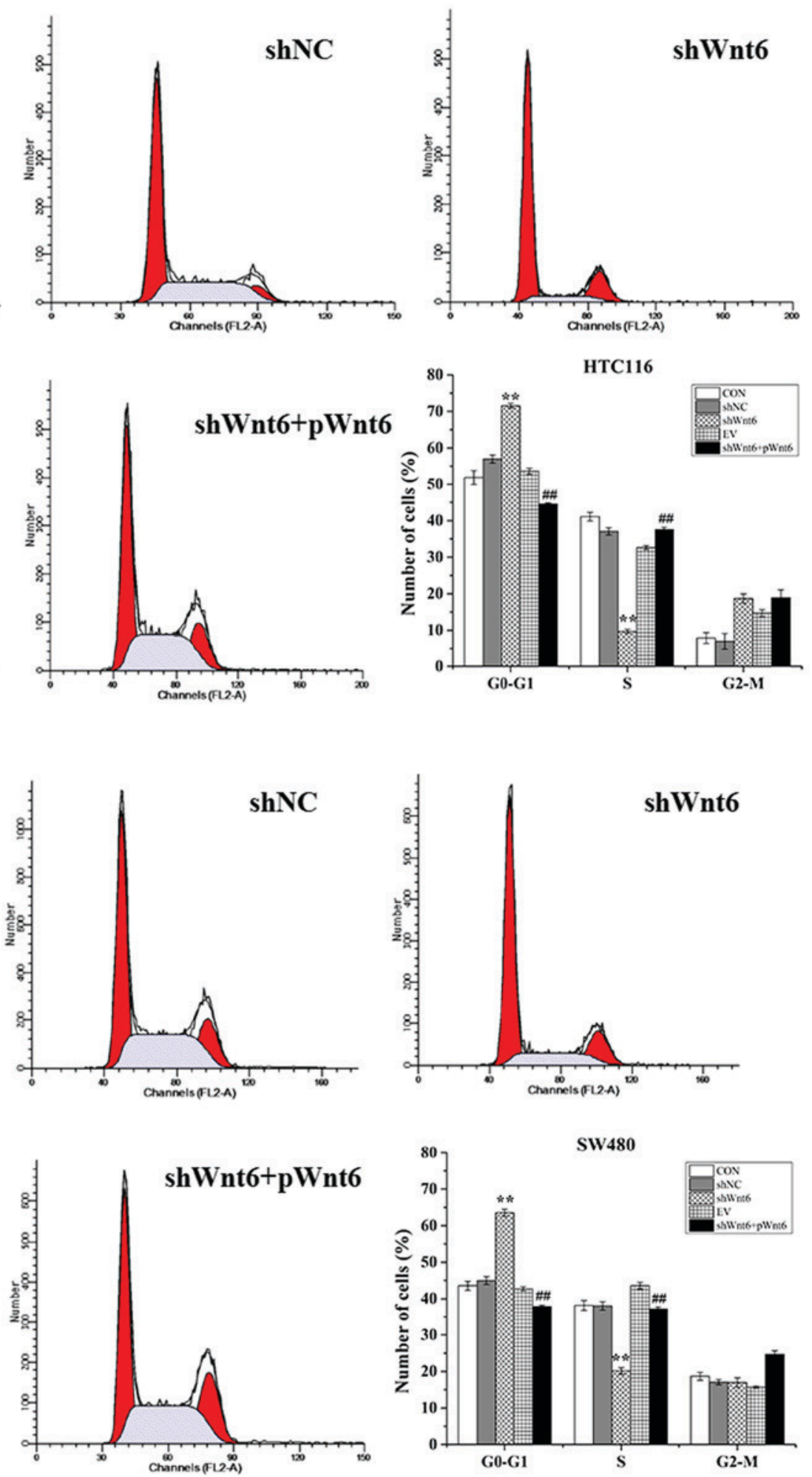

Figure 4. Inhibition of Wnt6 induces cell cycle arrest in $\mathrm{G}_{0}-\mathrm{G}_{1}$ phase. The cell cycle distribution in (A) HCT116 and (B) SW480 cells was evaluated using flow cytometry in CON, shNC, shWnt6, EV and shWnt6 groups. ${ }^{* *} \mathrm{P}<0.01 \mathrm{vs}$. shNC; ${ }^{\#} \mathrm{P}<0.01 \mathrm{vs}$. shWnt6. sh, short hairpin; NC, negative control; EV, empty vector; p, plasmid; CON, control.

differ in colon cell lines due to differences in histological differentiation, tumor staging and biological characteristics. Kirikoshi et al (15) reported that Wnt6 is strongly expressed in SW480 cells. Wnt proteins have been reported to promote various types of cancer. Wnt1 regulates the progression of breast cancer by promoting cell proliferation and migration (32).
Overexpression of Wnt 2 contributes to tumorigenesis of colorectal and lung cancer $(33,34)$. Wnt10b expression serves an important function in the development of endometrial cancer (35). In the present study, it was demonstrated that inhibition of Wnt6 may inhibit cell proliferation, cell cycle process and migration, and promote cell apoptosis, and overexpression 
A

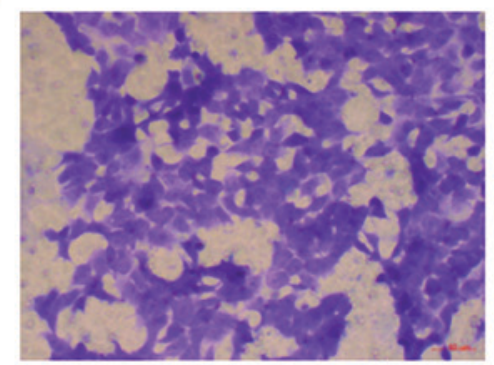

EV

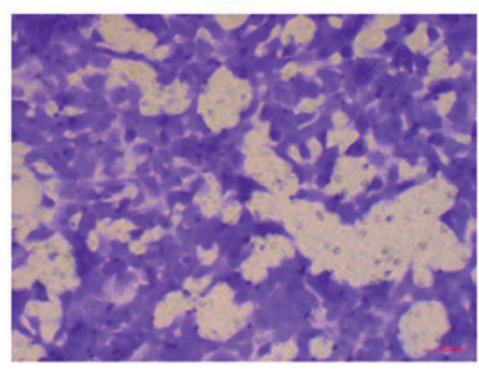

B

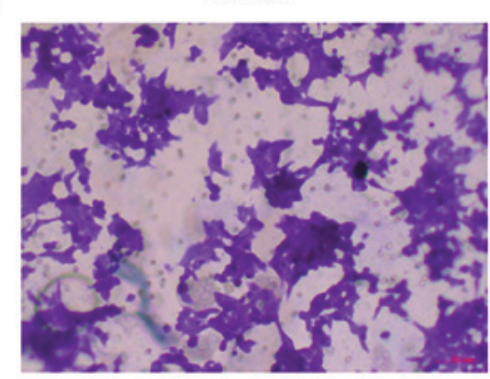

EV

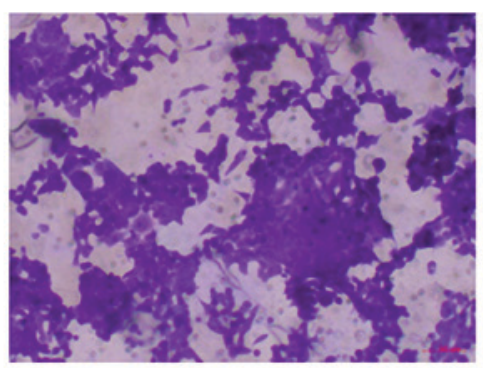

$\operatorname{shNC}$

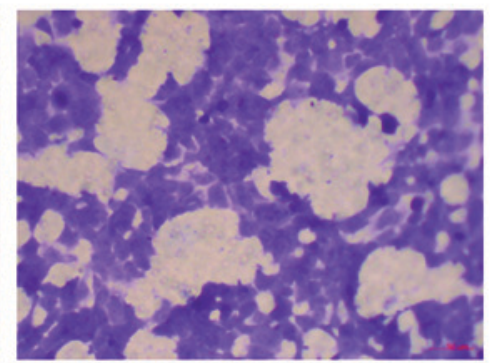

shWnt6+pWnt6
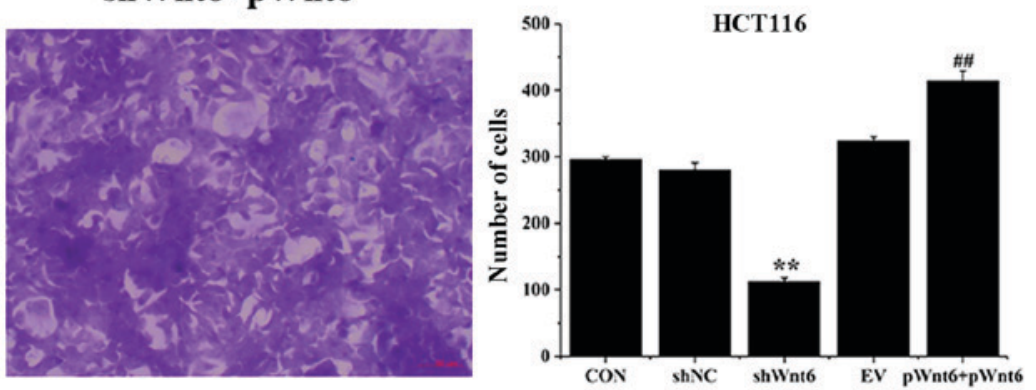

shWnt6

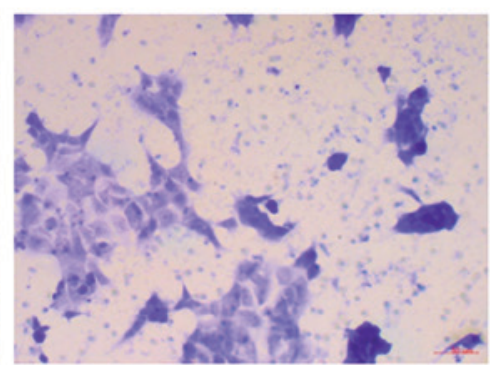

ov
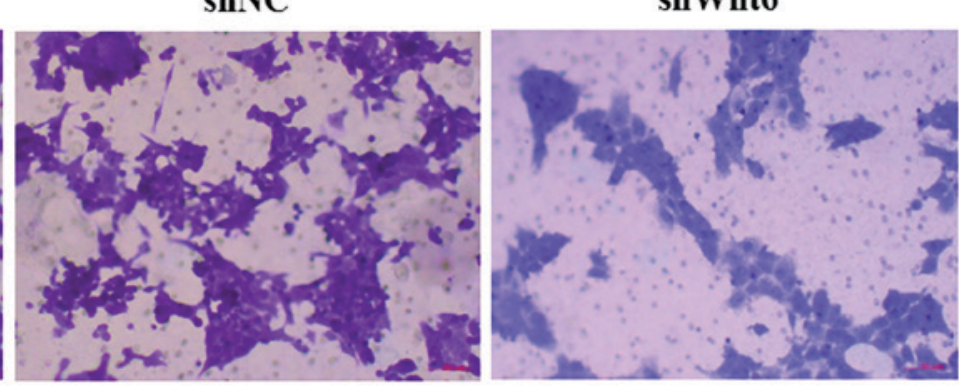

shWnt6+pWnt6
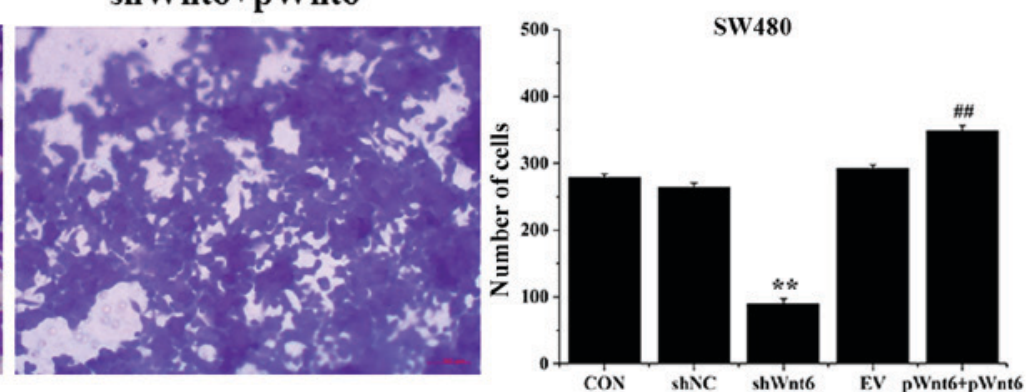

Figure 5. Wnt6 promotes the migration of HCT116 and SW480 cells. The migration of (A) HCT116 and (B) SW480 cells was evaluated using Transwell assays in CON, shNC, shWnt6, EV and shWnt6 groups. ${ }^{* *} \mathrm{P}<0.01$ vs. shNC; ${ }^{\# \#} \mathrm{P}<0.01$ vs. shWnt6. sh, short hairpin; NC, negative control; EV, empty vector; p, plasmid; CON, control.

of Wnt6 reversed this effect, thus upregulation of Wnt6 may contribute to tumorigenesis and development of malignant colon tumor.

The results demonstrated that the expression of caspase- 3 and MMP2 was increased, whereas the expression of Bax was decreased following overexpression of Wnt6. Caspase-3 has been demonstrated to be cleaved in apoptotic cells and the expression of caspase- 3 precursor decreased (36). The results of the present study demonstrated that overexpression of Wnt6 increased the expression of caspase-3 precursor, indicating that Wnt6 may inhibit cell apoptosis. MMP2 is involved in the breakdown of extracellular matrix. MMP2 is associated with the development of various malignant tumors and may promote EMT, a key process involved in cancer metastasis (37). Wnt6 increased the expression of MMP2 indicating that Wnt6 may promote cell migration. Bax is an apoptosis-promoting member of the Bcl-2 family (38). The results of the present study demonstrated that overexpression of Wnt6 decreased the expression of Bax, indicating that Wnt6 may inhibit cell apoptosis. Wnt1, Wnt3 and Wnt8 have been reported to activate $W n t / \beta$-catenin signaling pathway $(39,40)$. However, whether Wnt6 may activate the Wnt signaling pathway in colon cancer requires further investigation. 
A

\section{CON shNC shWnt6 EV shWnt6+pWnt6}

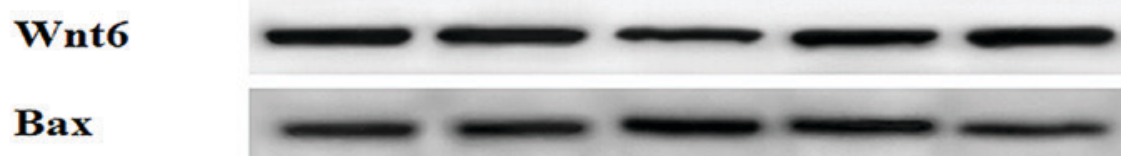

Caspase-3

MMP2

\section{及-actin}

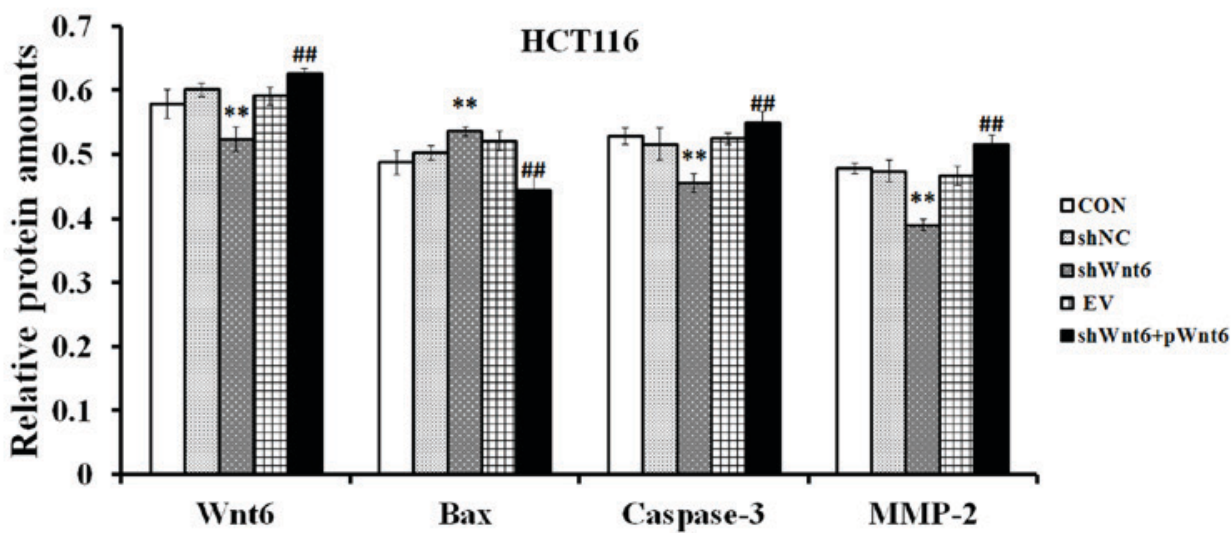

B CON shNC shWnt6 EV shWnt6+pWnt6

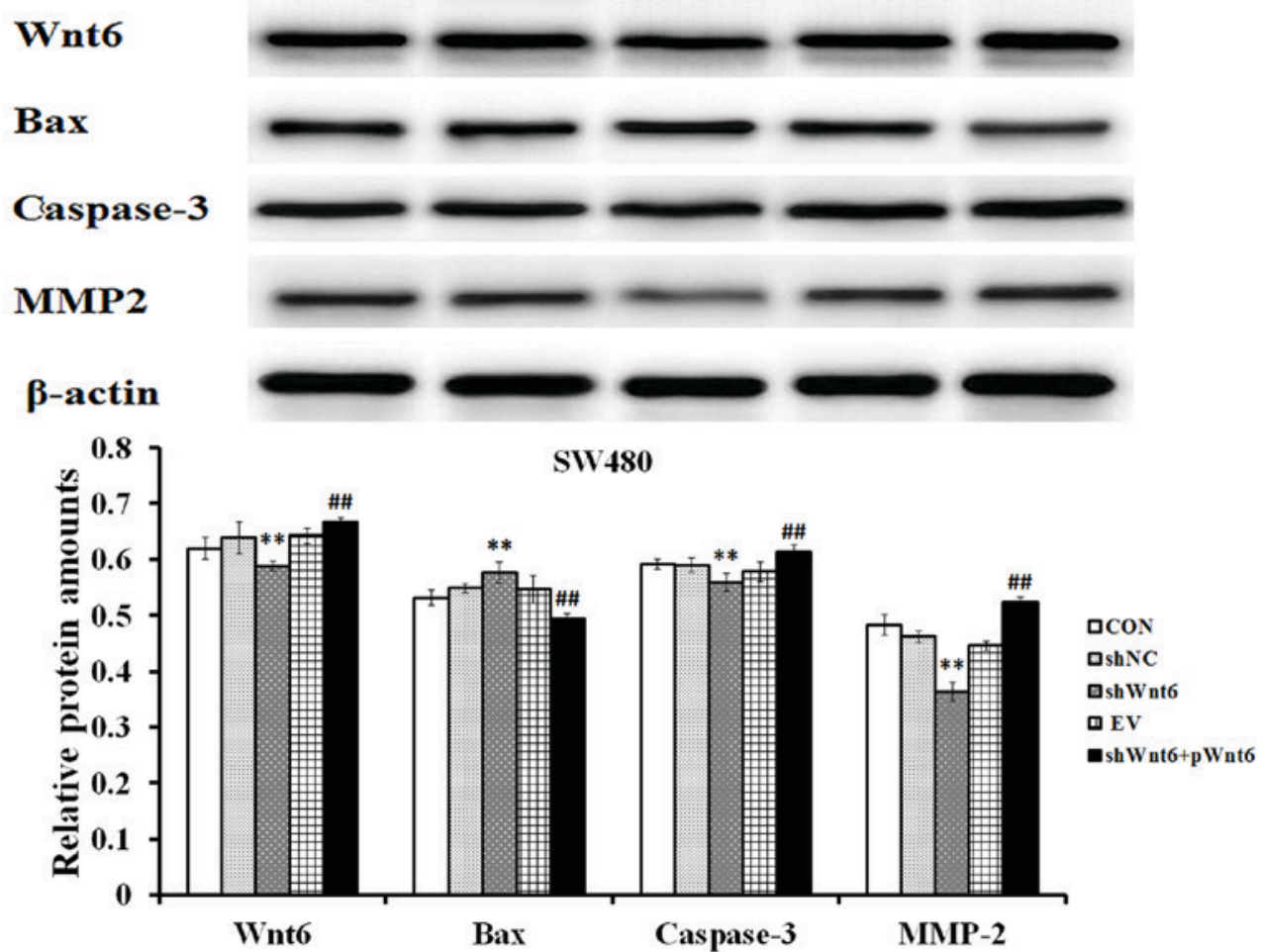

Figure 6. Wnt6 regulates the expression of Bax, caspase-3 and MMP2. The expression of Bax, caspase-3 and MMP2 in (A) HCT116 and (B) SW480 cells was evaluated using western blot analysis. ${ }^{* *} \mathrm{P}<0.01$ vs. shNC; ${ }^{\# \#} \mathrm{P}<0.01$ vs. shWnt6. sh, short hairpin; NC, negative control; EV, empty vector; p, plasmid; CON, control; Bax, B-cell lymphoma 2-associated X protein; MMP, matrix metalloproteinase.

The present study demonstrated that HCT116 and SW480 cells exhibit increased expression levels of Wnt6. Downregulation of Wnt6 by shWnt6 inhibited cell proliferation and migration and induced cell apoptosis of HCT16 and SW480 cells. Additionally, overexpression of Wnt6 may promote the proliferation, cell cycle and 
migration of HCT116 and SW480 cells, but inhibit cell apoptosis by upregulation of the expression of caspase- 3 and MMP2, and downregulation of the expression of Bax. These results indicated that Wnt6 may serve a vital function in the progression of colon cancer and may be utilized as a potential therapeutic target.

\section{Acknowledgements}

Not applicable.

\section{Funding}

The present work was supported by the National High Technology Research and Development Program 863 (grant no. 20151127D2811).

\section{Availability of data and materials}

All data generated or analyzed during this study are included in this published article.

\section{Authors' contributions}

$\mathrm{XZ}$ was responsible for all the experiments and data analyses and editing of the manuscript; HY was responsible for the overall design of the study and responsible for providing the materials. All the authors approved the final submission.

\section{Ethics approval and consent to participate}

Not applicable.

\section{Consent for publication}

Not applicable.

\section{Competing interests}

The authors declare that they have no competing interests.

\section{References}

1. Ahmed $\mathrm{S}$, Johnson $\mathrm{K}$, Ahmed $\mathrm{O}$ and Iqbal $\mathrm{N}$ : Advances in the management of colorectal cancer: From biology to treatment. Int J Colore Dis 29: 1031-1042, 2014.

2. László L: Predictive and prognostic factors in the complex treatment of patients with colorectal cancer. Magy Onkol 54: 383-394, 2010

3. Sung JJ, Lau JY, Goh KL and Leung WK; Asia Pacific Working Group on Colorectal Cancer: Increasing incidence of colorectal cancer in Asia: Implications for screening. Lancet Oncol 6: 871-876, 2005

4. $\mathrm{Hu} \mathrm{T}$, Li Z, Gao CY and Cho CH: Mechanisms of drug resistance in colon cancer and its therapeutic strategies. World J Gastroenterol 22: 6876-6889, 2016.

5. Shi W, Ye Z, Zhuang L, Li Y, Shuai W, Zuo Z, Mao X, Liu R, $\mathrm{Wu}$ J, Chen $\mathrm{S}$ and Huang W: Olfactomedin 1 negatively regulates NF-kB signalling and suppresses the growth and metastasis of colorectal cancer cells. J Pathol 240: 352-365, 2016.

6. Yan H, Hu K, Wu W, Li Y, Tian H, Chu Z, Koeffler HP and Yin D: Low expression of DYRK2 (Dual specificity tyrosine phosphorylation regulated kinase 2) correlates with poor prognosis in colorectal cancer. PLoS One 11: e0159954, 2016.
7. Zhang Y, Lin C, Liao G, Liu S, Ding J, Tang F, Wang Z, Liang X, Li B, Wei Y, et al: MicroRNA-506 suppresses tumor proliferation and metastasis in colon cancer by directly targeting the oncogene EZH2. Oncotarget 6: 32586-32601, 2015.

8. Zhou FQ, Qi YM, Xu H, Wang QY, Gao XS and Guo HG Expression of EpCAM and Wnt/ $\beta$-catenin in human colon cancer. Genet Mol Res 14: 4485-4494, 2015.

9. Phipps AI, Shi Q, Newcomb PA, Nelson GD, Sargent DJ, Alberts SR and Limburg PJ: Associations between cigarette smoking status and colon cancer prognosis among participants in North central cancer treatment group phase III trial N0147. J Clin Oncol 31: 2016-2023, 2013.

10. Clevers $\mathrm{H}$ and Nusse $\mathrm{R}$ : Wnt/ $\beta$-catenin signaling and disease. Cell 149: 1192-1205, 2012.

11. Luo J, Chen J, Deng ZL, Luo X, Song WX, Sharff KA, Tang N, Haydon RC, Luu $\mathrm{HH}$ and He TC: Wnt signaling and human diseases: What are the therapeutic implications? Lab Invest 87: 97-103, 2007.

12. Bienz $M$ and Clevers $H$ : Linking colorectal cancer to Wnt signaling. Cell 103: 311-320, 2000

13. Basu S, Haase G and Ben-Ze'ev A: Wnt signaling in cancer stem cells and colon cancer metastasis. F1000Res 5: pii: F1000 Faculty Rev-699, 2016.

14. Kikuchi A: Canonical Wnt signaling pathway and cellular responses. Clin Calcium 23: 799-807, 2013 (In Japanese)

15. Kirikoshi H, Sekihara H and Katoh M: WNT10A and WNT6, clustered in human chromosome $2 \mathrm{q} 35$ region with head-to-tail manner, are strongly coexpressed in SW480 cells. Biochem Biophys Res Commun 283: 798-805, 2001.

16. Yuan G, Regel I, Lian F, Friedrich T, Hitkova I, Hofheinz RD, Ströbel P, Langer R, Keller G, Röcken C, et al: WNT6 is a novel target gene of caveolin-1 promoting chemoresistance to epirubicin in human gastric cancer cells. Oncogene 32: 375-387, 2013.

17. Katoh M: WNT and FGF gene clusters (review). Int J Oncol 21: 1269-1273, 2002

18. Lavery DL, Davenport IR, Turnbull YD, Wheeler GN and Hoppler S: Wnt6 expression in epidermis and epithelial tissues during Xenopus organogenesis. Dev Dyn 237: 768-779, 2008.

19. Galbraith RL, Poole EM, Duggan D, Muehling J,Hsu L, Makar K, Xiao L, Potter JD and Ulrich CM: Polymorphisms in WNT6 and WNT10A and colorectal adenoma risk. Nutr Cancer 63: 558-564, 2011.

20. Livak KJ and Schmittgen TD: Analysis of relative gene expression data using real-time quantitative PCR and the 2(-Delta Delta C(T)) method. Methods 25: 402-408, 2001.

21. Na YJ, Jeon YJ, Suh JH, Kang JS, Yang KH and Kim HM: Suppression of IL-8 gene expression by radicicol is mediated through the inhibition of ERK1/2 and p38 signaling and negative regulation of NF-kappaB and AP-1. Int Immunopharmacol 1: 1877-1887, 2001.

22. Li X, Xu Y, Chen Y, Chen S, Jia X, Sun T, Liu Y, Li X, Xiang R and Li N: SOX2 promotes tumor metastasis by stimulating epithelial-to-mesenchymal transition via regulation of $\mathrm{WNT} / \beta$-catenin signal network. Cancer Lett 336: 379-389, 2013.

23. Lustig B and Behrens J: The Wnt signaling pathway and its role in tumor development. J Cancer Res Clin Oncol 129: 199-221, 2003.

24. Kirikoshi H, Sekihara H and Katoh M: Up-regulation of WNT10A by tumor necrosis factor alpha and Helicobacter pylori in gastric cancer. Int J Oncol 19: 533-536, 2001.

25. Mukherjee N, Bhattacharya N, Alam N, Roy A, Roychoudhury S and Panda CK: Subtype-specific alterations of the Wnt signaling pathway in breast cancer: Clinical and prognostic significance. Cancer Sci 103: 210-220, 2012.

26. Guillen-Ahlers H: Wnt signaling in renal cancer. Curr Drug Targets 9: 591-600, 2008.

27. Chen G, A J, Wang M, Farley S, Lee LY, Lee LC and Sawicki MP: Menin promotes the Wnt signaling pathway in pancreatic endocrine cells. Mol Cancer Res 6: 1894-1907, 2008.

28. Robinson DR, Zylstra CR and Williams BO: Wnt signaling and prostate cancer. Curr Drug Targets 9: 571-580, 2008.

29. Larue L and Delmas V: The WNT/Beta-catenin pathway in melanoma. Front Biosci 11: 733-742, 2006.

30. Modder UI, Oursler MJ, Khosla S and Monroe DG: Wnt10b activates the Wnt, notch, and NFkB pathways in U2OS osteosarcoma cells. J Cell Biochem 112: 1392-1402, 2011.

31. Yuan G, Regel I, Lian F, Friedrich T, Hitkova I, Hofheinz RD, Ströbel P, Langer R, Keller G, Röcken C, et al: WNT6 is a novel target gene of caveolin-1 promoting chemoresistance to epirubicin in human gastric cancer cells. Oncogene 32: 375-387, 2013. 
32. Wieczorek M, Paczkowska A, Guzenda P, Majorek M, Bednarek AK and Lamparska-Przybysz M: Silencing of Wnt-1 by siRNA induces apoptosis of MCF-7 human breast cancer cells. Cancer Biol Ther 7: 268-274, 2008.

33. Jung YS, Jun S, Lee SH, Sharma A and Park JI: Wnt 2 complements Wnt/ $\beta$-catenin signaling in colorectal cancer. Oncotarget 6: 37257-37268, 2015.

34. Huang C, Ma R, Xu Y, Li N, Li Z, Yue J, Li H, Guo Y and Qi D: Wnt2 promotes non-small cell lung cancer progression by activating WNT/ $\beta$-catenin pathway. Am J Cancer Res 5: 1032-1046, 2015

35. Chen H, Wang Y and Xue F: Expression and the clinical significance of Wnt 10a and Wnt10b in endometrial cancer are associated with the Wnt/ $\beta$-catenin pathway. Oncol Rep 29: 507-514, 2013.

36. Cao LH, Li HT, Lin WQ, Tan HY, Xie L, Zhong ZJ and Zhou JH: Morphine, a potential antagonist of cisplatin cytotoxicity, inhibits cisplatin-induced apoptosis and suppression of tumor growth in nasopharyngeal carcinoma xenografts. Sci Rep 6: 18706, 2016.
37. Gialeli C, Theocharis AD and Karamanos NK: Roles of matrix metalloproteinases in cancer progression and their pharmacological targeting. FEBS J 278: 16-27, 2011.

38. Sugimoto C, Fujieda S, Seki M, Sunaga H, Fan GK, Tsuzuki H, Borner C, Saito H and Matsukawa S: Apoptosis-promoting gene (bax) transfer potentiates sensitivity of squamous cell carcinoma to cisplatin in vitro and in vivo. Int J Cancer 82: 860-867, 1999.

39. Lee EH, Chari R, Lam A, Ng RT, Yee J, English J, Evans KG, Macaulay C, Lam S and Lam WL: Disruption of the Non-canonical WNT pathway in lung squamous cell carcinoma. Clin Med Oncol 2: 169-179, 2008.

40. Ramel MC and Lekven AC: Repression of the vertebrate organizer by Wnt 8 is mediated by Vent and Vox. Development 131 : 3991-4000, 2004. 